\title{
ATTITUDES TO THE APPLICATION IN PRACTICE OF THE SPORTS- ENTERTAINMENT COMPETENCE OF THE EDUCATOR
}

\author{
Donka Zheleva-Terzieva ${ }^{1}$
}

\begin{abstract}
:
Introduction: This article examines and analyses the need for the formation of professional-pedagogical competence for the realization of sports-animation activities in an educational environment. The opinion of current pedagogues and competent persons (experts in the field of physical education, sports animation and pedagogical area) have been studied, as well as the change in the attitudes of students from pedagogical specialties, as a result of training, on the application of the sportsanimation competence of the pedagogy in and out of professional pedagogical practice.

Methods: The empirical method used is a survey applied to 291 persons. The results obtained are subject to processing and statistical analysis by the methods of quantitative analysis of survey data - Van der Waerden test and Z-test. The concordance coefficient has been calculated - to determine the degree of consistency in the expert assessment and the main empirical characteristic, - as a well as the arithmetic mean.

Findings and results: The results show high values of the indicators characterizing the opinions and attitudes of the surveyed persons both in the direct professional-pedagogical activity and on its realization beyond the vocational-pedagogical practice (within leisure time) and confirm the existence of grounds and real needs from the formation of professional-pedagogical competence for the implementation of sports-animation activities in an educational environment.
\end{abstract}

UDC Classification: 378, DOI: https://doi.org/10.12955/pss.v2.246

Keywords: attitudes, application, pedagogical competence, sports animation.

\section{Introduction}

Motor activity in modern society is mainly achieved through physical culture. Research by UNESCO and other scientific institutes proves that the use of sport as one of the components of physical culture is a prerequisite for a healthy lifestyle. Sport is attractive to people because of the psycho-emotional environment, where different types of activities take place. The role of sport is important for the physical and mental health of people, including children. The social environment - parents and teachers - plays a major role in promoting sports interests in children with the objective to optimize the motor regime of adolescents (Tomova et al., 2015).

Thise article focuses on sports animation in an institutionally organized environment as a form of pedagogical activity aimed at meeting children's needs for recreation, motor and entertainment interests while organizing leisure time in and out of the system of educational institutions. The purpose of sports-animation activity when working with children is to achieve fun, pleasure, positive emotions and mental relief from the educative work, to improve motor qualities, skills and physical capacity, and to increase the body's resistance to external influences. To organize and conduct sports-animation activities with children, the educators need to have the appropriate competence. "For the successful implementation of sports and entertainment activities, specialists in the field of sports-entertainment services are required to have good basic training, high professional competence and realization with creativity, responsibility and the pursuit of continuous self-improvement" (Tsankov, 2013).

D. Zheleva-Terzieva (2019) brings up the topicality of the educator`s sports-animation competence, prompted by:

- the insufficient level of motor activity of people;

- the need for alternative forms of activity to meet the generally accepted norms of motor activity to maintain good health;

- the existing extracurricular forms of physical education activity are not mandatory for children, and the surrounding world (computer, tablet, telephone and related internet, social networks, etc.) provides many other interesting activities;

- the reality is that the class leader is the attendant of the organized tourist events and student camps, rather than the physical education and sports teacher, a sports coach or a sports educator;

\footnotetext{
${ }^{1}$ Trakia University, Faculty of Education, donkazeleva@ gmail.com
} 
- sports animators can be found mainly in expensive resorts and luxury hotels and at that, not all year round;

- the ability of the educator to apply acquired competence beyond his/ her professional duties, which could be used to fill deficits in this regard.

The main objective of education and the professional work of teachers as representatives of the most widely applicable pedagogical profession is concentrated in creating conditions for the full development of children, including physical (motor) development (Schivacheva-Pineda, 2005). "The profession of the teacher is the most intensively developing profession, it continuously faces challenges related to the different aspects of the evolving global and information society" (Neminska, 2019).

The National Strategy for the Development of Physical Education and Sports in the Republic of Bulgaria defines as important the tasks for changing the organization of extracurricular and extraschool sports activities and organizing sports activities during school holidays as an effective means against existing social deformities among adolescents. Not only physical education specialists, but also all educators need to have competence to organize and conduct sports-animation activities in the real conditions of the natural environment and in the full-time mode of study of educational institutions.

The high quality of the teacher's professional activity is reflexively referenced to the rules clearly set out in the respective profession-graph for the successful selection of those wishing to devote themselves to the teaching profession, academic and practical training which are, adequate to the modern requirements, and the permanent improvement of the professional qualification of teachers (Teneva, 2018). The professional choice of students is tied to the needs, abilities and interests of the individual (Teneva, 2017).

When considering university students learning motivation, account should be taken of the higher level of development of consciousness and awareness of the learning activity compared to that of school students. For university students, participation in the learning process is voluntary in nature, although the school student`s unconscious approach in specific situations is maintained.

Attitudes towards learning, and in particular the preparation for a particular discipline, have its varieties, and can be characterized by maturity, responsibility, interest, motivation, positive attitudes towards the upcoming professional and personal realization (Schivacheva-Pineda, 2012).

Attitudes are also accepted as part of an analytical framework consisting of: concept, approach and belief. The concept represents the highest level of thinking and is related to learners' views on objects and processes of learning. Attitudes present learners' ideas about concept-based learning objects and processes (Benson \& Lor, 1999). Building relationships and value positions to the developed topics and problems is a fundamental part of the positive value attitude (Temnikova, 2018).

The participation of students in vocational training forms naturally raises its expectations for new ways of pedagogical preparation and the renewal of instruments in accordance with the requirements of modern technologies (Petrova, 2004).

In this study, the interest of the author is directed to the mindset and attitudes of four groups of persons familiar with and relevant to the educational discipline "Sports animation in an educational environment" in the pedagogical specializations of the Trakia University - Stara Zagora.

\section{Methodology}

The above-mentioned school discipline was introduced in the forms of an elective and the optional preparation of the specializations "Pre-school and primary school pedagogy", "Primary school pedagogy with a foreign language" and "Social pedagogy" for the "Bachelor" EQD and "Master" EQD as well as for the postgraduate qualification courses "Children's teacher" and "Primary school teacher" from the 2017/2018 academic year. The research period is August 2017 - January 2021, during which 193 students and 38 post-graduate students were involved in the training. With each of the two groups, immediately before and after the training course, a standardized written survey with closed scaled responses was conducted. Between January and March 2020, an online survey with 51 active educators was conducted, and in 2018 an internet communication with 9 competent persons - experts in the field of physical education, sports animation and pedagogical area was performed. The contingent of this study is 291 persons and it aims at studying the attitudes of undergraduate students 
and post-graduate students and the opinion of the current educators and experts on the applicability of the sports-animation competence formed within the professional-pedagogical activity.

To achieve the objective, an empirical method was used and, applied in two variants - in paper and electronic form through the questions:

Question 1. Do you consider that the competence formed in the educational discipline "Sports animation in an educational environment" will be useful for the professional realization of the educator?

Question 2. Do you consider that basic preparation in sports animation would be useful for the personal development and public activity of educators outside of their professional activity?

Figure 1 presents the results of the applied survey from 231 learners (193 students and 38 postgraduate students) before the training process.

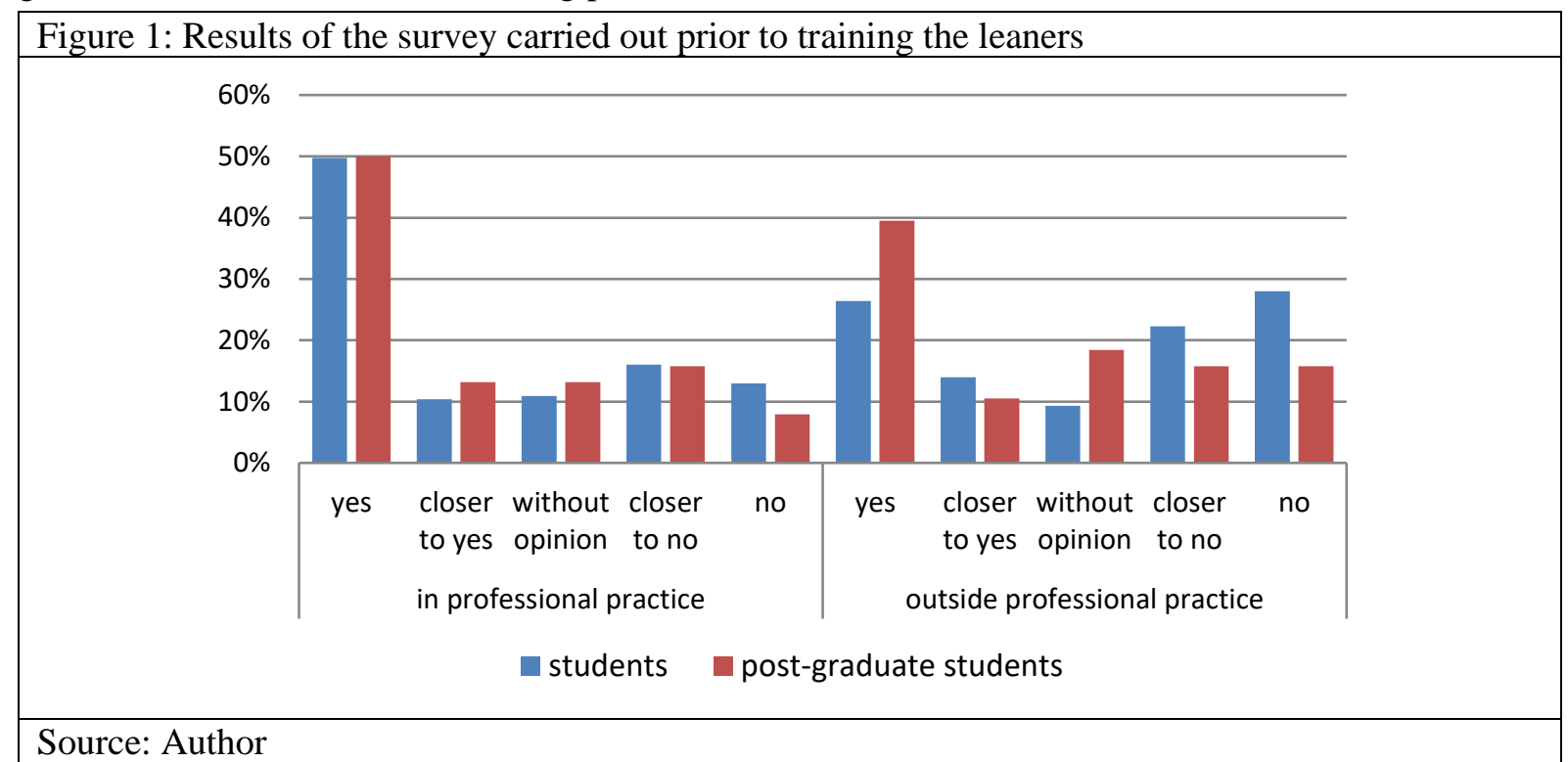

The results show that $60.11 \%$ of students and $63.16 \%$ of post-graduate students see benefits from the vocational-pedagogical training and $40.42 \%$ students and $50 \%$ post-graduate students respectively see benefit outside the pedagogical sphere. Negative attitudes are significantly more pronounced among students $(-29.01 \%)$ on the applicability of sports-animation competence in vocationalpedagogical activity and $(50.27 \%)$ - outside the professional duties. The proportion of respondents who did not have an opinion on the questions asked ranged from 9.33 to $10.88 \%$ for undergraduate students and from 13.16 to $18.42 \%$ for post-graduate students.

\begin{tabular}{|l|l|l|l|l|}
\hline Figure 2: Results of the survey carried out after training of the leaners \\
\hline $60 \%$ & & & \\
\hline $50 \%$ & & & \\
\hline $40 \%$
\end{tabular}


The answers to the questions of the survey, conducted immediately after the completion of the course, in the attitudes of the students there is a slight decrease $-57.51 \%$ and $39.83 \%$ respectively for the application of the considered competence in and outside the pedagogical sphere. For post-graduate students, the result was the same. It can be concluded that after the completion of the training process, the share of negative attitudes, although minimally has decreased. The results are presented in Figure 2.

The Z-test was used for the quantitative analysis of the survey data, the application of which is not affected by the volume of samples (Kozhuharova et al., 2012).

Formulated were the following zero and alternative hypotheses:

$\mathrm{H}_{0}$ : the difference in relative frequencies between the two samples (before and after the training) is not statistically significant, i.e., it is due to random factors;

- $\quad \mathrm{H}_{1}$ : the difference in relative frequencies between the two samples is statistically significant, i.e., it is due to the influence of the surveyed factor (the learning process).

With a probability of error $\alpha=0.07$ the "critical" value for $Z$ is $Z_{\alpha}=1.9954$.

The information necessary to determine $\mathrm{Z}_{\mathrm{em}}$ 's empirical characteristic of the hypothesis is presented in Table 1 .

\begin{tabular}{|l|l|l|r|}
\hline Table 1: Characteristics of the distribution of values in both studies \\
\hline & Mean & Std. deviation & Std. Error Mean \\
\hline Before training & 3.3377 & 1.58991 & 0.07397 \\
\hline After training & 3.3485 & 1.55931 & 0.07255 \\
\hline
\end{tabular}

Source: Author

The values of the resulting inequality $\mathrm{Z}_{\mathrm{em}}=-1.000<\mathrm{Z}_{\alpha}=1.9954$ show that the zero hypothesis $\mathrm{H}_{0}$ is correct, which is reason to conclude that the training carried out in the educational discipline "Sports animation in an educational environment" practically does not affect the attitudes of the learners to the applicability of the competences formed in and out of their future professional activity as educators.

Figure 3 presents the results of the survey of the opinion and attitude of 51 active educators and 9 experts.

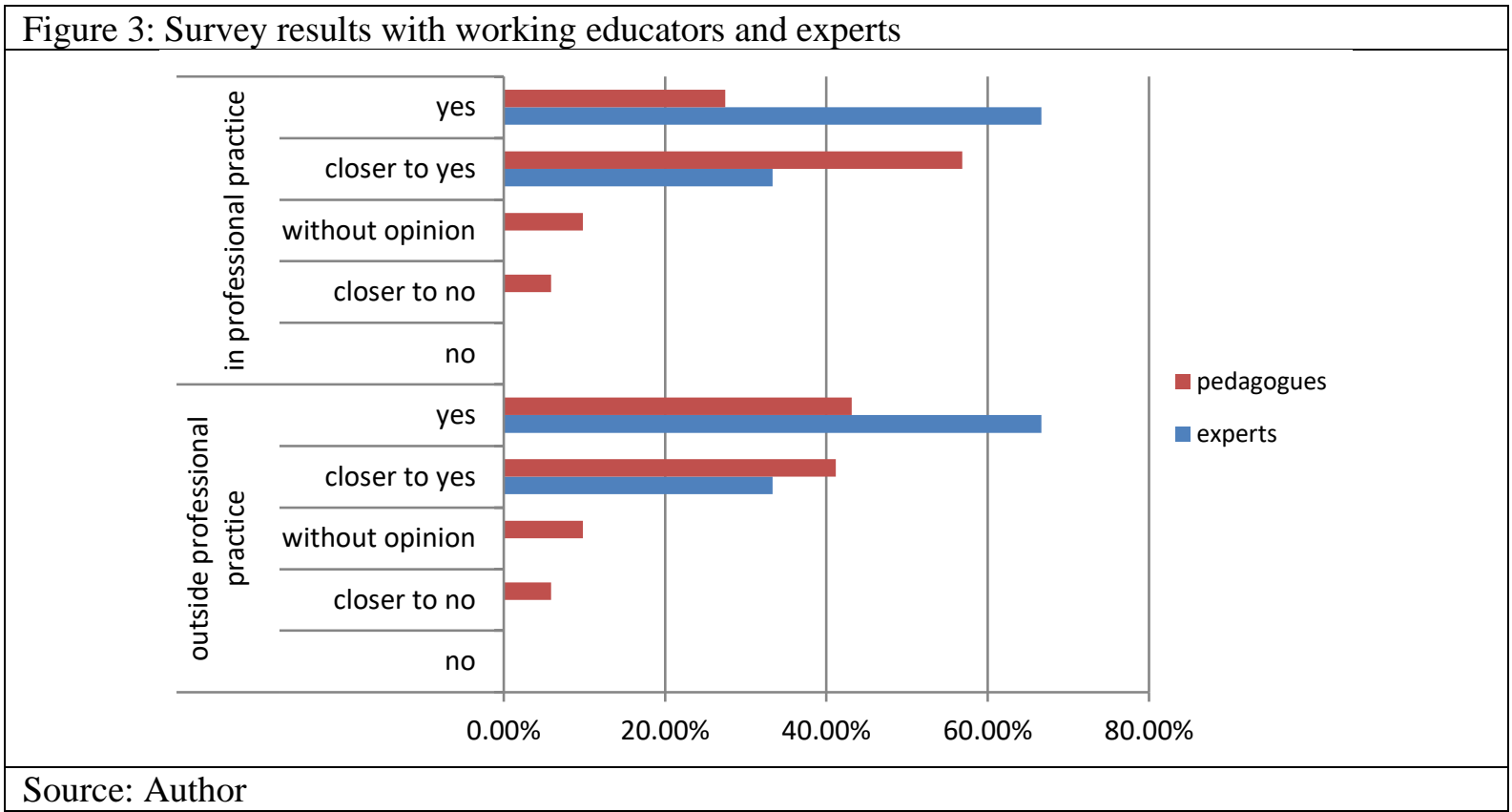

The data shows a high degree of positive opinion of the surveyed persons to the applicability of sportsanimation competence in and outside the professional activity of the educator. Almost negligible is the proportion of active educators who have indicated a negative or neutral answer to the issues studied, and for the experts - there are no negative or neutral answers. 
The degree of consistency between the opinions of the experts is determined by the coefficient of coordination, the value of which is close to one in this case $(\mathrm{W}=0.93)$. This indicates a high degree of coincidence of the opinions of the different experts.

\section{Conclusion}

The results of the survey show the influence of training on the attitudes of different groups of respondents, as well as the opinion of the two groups of specialists in the field:

- the expectations of students for the applicability of the sports-animation competence formed, both in the framework of direct professional-pedagogical activity and on the realization of such activity outside of vocational-pedagogical practice (within leisure time) are rather positive, with differences in attitudes before and after training showing are insignificant. In contrast, the attitudes of post-graduate students hardly changed. It can be assumed that the students do not yet have a real vision for their forthcoming professional activity and are therefore not so adamant in their assessments;

- the attitude of the experts as well as that of the working educators to the application of professional-pedagogical competence for sports-animation activity is entirely positive, both within and outside the pedagogical sphere.

\section{References}

Benson, P., W. Lor (1999). Conceptions of language and language learning. System, 27, 459-472.

Kozhuharova, G., Ganchev, G., Delchev, M. (2012). Methodology of pedagogical research for students and teachers. Retrieved from www.edu.uni-sz.bg

Nemisnka, R. (2019). For the profession of teacher through the prism of normativeness, theory and operationality. $E$ magazine "Pedagogical Forum", 1, 3-9.

Petrova, V. (2004). Expectations for renovation of the instruments for the formation of pedagogical abilities. Pedagogical Almanac, University Publishing House Sv. Sv. Kiril and Metodiy, 12 (1), 145-167.

Schivacheva-Pineda, I. (2005). The student and the teacher in the modern educational realities. Yambol.

Shivacheva-Pineda, I. (2012). Manifestations of students' motivation for learning. Announcements of Union of Scientists Sliven, 21, 162-167.

Temnikova, M. (2018). Strategies in mathematics training 1-4. Class. Stara Zagora.

Teneva, M. (2017). Who's a good teacher? Stara Zagora: Trakia University, Faculty of Education.

Teneva, M. (2018). Trends in the preparation and qualification of the modern Bulgarian teacher. Yearbook of the Faculty of Education, XV, 8-31.

The National Strategy for the development of Physical Education and Sport in the Republic of Bulgaria $2012-2022$, adopted by the NA on 24.11.2011. Retrieved from https//:mpes.government.bg

Tomova, S., V. Tsekov, D. Dimitrov (2015). Sports animation. Varna: UPH " Science and Economics".

Tsankov, N. (2013). The Competence Approach in the Cultural and-Pedagogical Field of Physical Education and Sports. Activities in physical education and sport, 3 (2), 209-214.

UNESCO (2016). Education for World Citizenship Prepare the Students for the Challenges of the 21st Century. Paris: Organization of the United Nations Education, the Science and Culture. Retrieved from https://unesdoc.unesco.org

Zheleva-Terzieva, D. (2019). Relevance and importance of the pedagogue`s sports-animation competence. E-magazine "Pedagogical Forum", 1, 51-57. 